\title{
Symmetric Fermion Mass Generation as Deconfined Quantum Criticality
}

\author{
Yi-Zhuang You, ${ }^{1}$ Yin-Chen $\mathrm{He},{ }^{1}$ Cenke $\mathrm{Xu},{ }^{2}$ and Ashvin Vishwanath ${ }^{1}$ \\ ${ }^{1}$ Department of Physics, Harvard University, Cambridge, Massachusetts 02138, USA \\ ${ }^{2}$ Department of Physics, University of California, Santa Barbara, California 93106, USA
}

(Received 28 September 2017; revised manuscript received 13 December 2017; published 14 February 2018)

\begin{abstract}
Massless $(2+1)$ D Dirac fermions arise in a variety of systems from graphene to the surfaces of topological insulators, where generating a mass is typically associated with breaking a symmetry. However, with strong interactions, a symmetric gapped phase can arise for multiples of eight Dirac fermions. A continuous quantum phase transition from the massless Dirac phase to this massive phase, which we term symmetric mass generation, is necessarily beyond the Landau paradigm and is hard to describe even at the conceptual level. Nevertheless, such transition has been consistently observed in several numerical studies recently. Here, we propose a theory for the symmetric mass generation transition which is reminiscent of deconfined criticality and involves emergent non-Abelian gauge fields coupled both to Dirac fermions and to critical Higgs bosons. We motivate the theory using an explicit parton construction and discuss predictions for numerics. Additionally, we show that the fermion Green's function is expected to undergo a zero-to-pole transition across the critical point.
\end{abstract}

DOI: 10.1103/PhysRevX.8.011026

Subject Areas: Condensed Matter Physics, Strongly Correlated Materials, Topological Insulators

\section{INTRODUCTION}

Recently, much attention has been lavished on band structures with symmetry protected nodal points (Dirac and Weyl semimetals) [1-7] in both two [8-10] and three spatial dimensions [11-15]. The paradigmatic example is graphene, where the band touching points are protected by symmetry, and the low-energy dispersion around these points is captured by the massless 2D Dirac equation [16]. Similarly, massless Dirac fermions also appear on the surface of free-fermion topological phases [17]. A key question pertains to the stability of the Dirac nodes in the presence of interactions. This controls whether the material remains a semimetal or develops a gap leading to a semiconductor. Typically, this has been discussed in terms of interaction-induced symmetry lowering, where interactions lead to a spontaneous symmetry breaking. The resulting lowering of symmetry allows for an energy gap. The physics in these settings can be modeled by a meanfield "mass" term that is spontaneously generated on lowering the symmetry, and gaps out the Dirac fermions. This is the standard mass generation in the Gross-Neveu [18] and the Yukawa-Higgs models. The main challenge

Published by the American Physical Society under the terms of the Creative Commons Attribution 4.0 International license. Further distribution of this work must maintain attribution to the author(s) and the published article's title, journal citation, and DOI. then is identifying the appropriate channel of symmetry breaking, following which one can utilize the Landau paradigm of order parameters to describe the mass generation.

In this work, we discuss an altogether different mechanism of mass generation for Dirac fermions, which breaks no symmetries and cannot be modeled by a single-particle mass term at the free-fermion level. The possibility of such a scenario is informed by recent developments in the theory of interacting fermionic symmetry protected topological (SPT) phases [19-32], relating to the stability of freefermion topological insulators or superconductors to interactions. The paradigmatic example given by Fidkowski and Kitaev $[19,20]$ is the $(1+1)$ D Majorana chain with an appropriately defined time reversal that protects edge Majorana modes regardless of their multiplicity. However, interactions lead to an energy gap to these modes when they are multiples of eight, leading to a reduction of the freefermion classification $\mathbb{Z} \rightarrow \mathbb{Z}_{8}$. More relevant to our purposes is the interaction reduction of $(2+1) \mathrm{D}$ surface states of $(3+1) \mathrm{D}$ topological phases, which contain Majorana or Dirac fermions. Indeed, here with the standard time reversal for electrons (class DIII) [24,25,33], there is an interaction reduced classification $\mathbb{Z} \rightarrow \mathbb{Z}_{16}$ of topological superconductors, implying that 16 surface Majorana fermions or equivalently eight Dirac fermions are unstable towards a massive (gapped) phase in the presence of strong interactions without breaking any symmetry.

These considerations prompt us to look for a model of an electronic semimetal with eight Dirac nodes in 2D. A single 
layer of graphene with its twofold valley and twofold spin degeneracy leads to four Dirac nodes; hence, we need to consider two layers [34] of graphene to obtain eight Dirac cones in all (by combining the valley, spin, and layer degeneracies). There is a simple way to see that at half filling it is possible to realize a symmetric insulating phase if interactions are included. Let us consider an antiferromagnetic Heisenberg spin interaction $H_{\text {int }}=J \sum_{i} S_{i 1} \cdot S_{i 2}$ between the vertically displaced sites across the two layers [35]. Since on average we have one electron per site and each electron carries spin- $1 / 2$, the two electrons across the layer will pair into singlets and acquire an energy gap as long as the interlayer Heisenberg interaction is strong enough. This leads to a fully gapped and nondegenerated ground state, which can be described as a direct product state of interlayer singlets. The state neither breaks any symmetry nor does it develop topological order. Therefore, it is a featureless gapped phase in $(2+1) \mathrm{D}$ [36-38]. The strong-coupling interaction mass (the many-body gap) that the electrons acquire in this phase is called the symmetric mass [39], and the continuous phase transition (if it exists) between the Dirac semimetal and the featureless insulator will be called symmetric mass generation (SMG) [40-42]. Note that one can also discuss the transition for a system with fewer Dirac fermions. For the surface of a fermionic SPT phase (e.g., in class DIII or AIII), the gapped phases necessarily involve topological order $[24,25,33]$ and constitute a rather different problem. For the intrinsically $2 \mathrm{D}$ system of graphene with an even number of sites in the unit cell, it is believed there is no intrinsic obstruction to realizing a trivial gapped phase (e.g., as shown for spinful single layer graphene in Ref. [38]). However, writing a Hamiltonian that realizes these gapped phases is itself a nontrivial task. Therefore, we focus on the case of eight Dirac nodes in $(2+1) D$ systems where the gapped phases are readily accessible and the numerical evidence for a single continuous transition is encouraging.

What are the possible scenarios for the transition from the Dirac semimetal to the featureless gapped phase? At least for small $J$, it is known that short-ranged interactions are perturbatively irrelevant for 2D Dirac fermions; thus, the transition can occur only at finite interaction strengths. Unlike the lower-dimensional cases, where the instability of $(1+1) \mathrm{D}$ gapless fermions is manifest perturbatively and/or can be studied with powerful tools such as bosonization, the situation for the $(2+1) \mathrm{D}$ problem is more challenging. On general grounds, there could be several scenarios as we step out of the Dirac semimetal phase. First, there could simply be a direct first-order transition to the featureless gapped phase, where the symmetric mass gap opens up discontinuously. Next, an intervening symmetry breaking phase may occur, leading to an energy gap to the fermions. Subsequently, the symmetry could be restored, accomplishing the phase change in a two-step process, as illustrated in Fig. 1(a). A different two-step
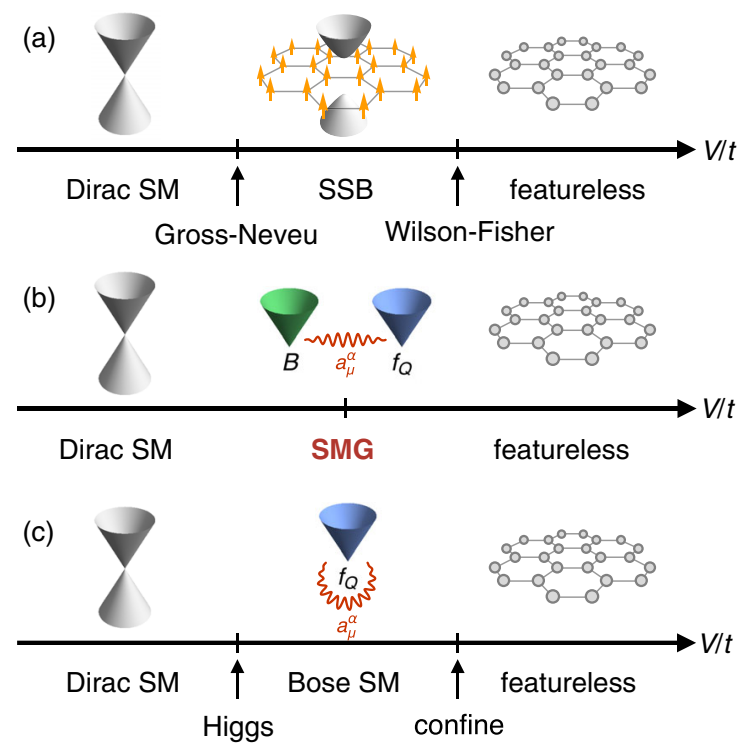

FIG. 1. Possible scenarios of transitions from the Dirac semimetal (Dirac SM) to featureless gapped phase. (a) Landau paradigm: an intermediate spontaneous symmetry breaking (SSB) phase sandwiched between the Gross-Neveu and the Wilson-Fisher transitions. (b) A direct continuous transition: the symmetric mass generation (SMG) as a deconfined quantum critical point, with emergent gauge field and fractionalized partons. (c) More exotic (and less likely) scenario: an intermediate Bose semimetal (Bose SM) critical phase between the Higgs and confinement transitions.

evolution involves the existence of an exotic critical phase that can be stable over a range of parameters, dubbed as the Bose semimetal phase [43-46] in Fig. 1(c). It is a gapless quantum liquid of bosons and can be described as a generalized Gutzwiller projected Dirac semimetal. The most interesting possibility is shown in Fig. 1(b), where the SMG occurs as a single continuous transition without any intermediate phases. Remarkably, numerical simulations of the problem in different models with various microscopic symmetries using different numerical methods $[35,40-42,47-49]$ seem to uniformly point towards a single continuous SMG transition. All these models share one key common property that the weakly interacting semimetal phase should have exactly eight massless Dirac fermions. Even at the conceptual level it is unclear how to write down a theory for this putative transition. This is the problem addressed in this work.

Since the SMG transition lies outside the Landau symmetry breaking paradigm, it would necessarily be exotic and require new ideas. The strategy we adopt is to consider a form of fractionalization, where the symmetry quantum number of the electron is peeled off from their Dirac dispersion and carried away by a set of bosonic partons, while the Dirac cone structure is still maintained by a set of symmetry neutral fermionic partons. The process of fractionalization leads to an emergent gauge interaction 
between the bosonic and fermionic partons. In this framework, the semimetal phase corresponds to the condensed (Higgs) phase of the bosons. The featureless gapped phase corresponds to the symmetric gapped phase of the bosons, which triggers gauge confinement of the remaining degrees of freedoms. This theory of SMG therefore falls in the category of deconfined quantum critical points [50-52] that contains a non-Abelian (Yang-Mills) gauge field coupled to both the massless scalar (Higgs) fields and eight flavors of massless Dirac fermions.

In the following, we first introduce a minimal model for the SMG in 2D with SU(4) symmetry. We develop an intuitive picture of the gapped phase as a paired superconductor in which fluctuations restore symmetry but leave the gap intact. This motivates our parton construction and lead to a field theory description for SMG. Finally, we discuss the implication of our theory for the fermion Green's function which can be tested in numerics.

\section{MODEL}

Consider the honeycomb lattice with four flavors of fermions at half filling on each site. This is a model of two layers of graphene (each with two component spinful fermions). Previously we discussed how an interlayer spin interaction could lead to singlets, but it will be useful to enhance the symmetry and consider the fermions to be fully symmetric under the SU(4) rotation of the four flavors. A minimal model that captures this is given by

$$
\begin{aligned}
H & =H_{0}+H_{I}, \\
H_{0} & =-t \sum_{\langle i j\rangle} \sum_{a=1}^{4}\left(c_{i a}^{\dagger} c_{j a}+\text { H.c. }\right),
\end{aligned}
$$

where $c_{i a}$ is the fermion operator on site $i$ and of flavor $a=1,2,3,4$. Now consider the interaction term which preserves SU(4) symmetry:

$$
H_{I}=-V \sum_{i}\left(c_{i 1}^{\dagger} c_{i 2}^{\dagger} c_{i 3}^{\dagger} c_{i 4}^{\dagger}+\text { H.c. }\right) \text {. }
$$

Note, however, the $V$ term does not preserve the charge conservation of the fermions. The charge $\mathrm{U}(1)$ symmetry is explicitly broken at the Hamiltonian level and excluded from our symmetry consideration. This may be interpreted as a proximity-induced charge- $4 e$ superconductivity (SC) [53-58]. If the regular (charge-2e) superconductivity was brought in proximity to graphene, the fermions would immediately be gapped. In contrast, the presence of weak four fermion terms $V \ll t$ does not destabilize the Dirac cone and a finite interaction strength is needed for a transition to occur. On the other hand, in the strongcoupling limit $V \gg t$, the ground state is a simple product state of on-site fluctuating charge- $4 e$ quartets:

$$
\left|\Psi_{c}\right\rangle=\prod_{i}\left(1+c_{i 1}^{\dagger} c_{i 2}^{\dagger} c_{i 3}^{\dagger} c_{i 4}^{\dagger}\right)\left|0_{c}\right\rangle,
$$

where $\left|0_{c}\right\rangle$ denotes the fermion vacuum state. Therefore, a transition is expected between the gapless Dirac semimetal and the gapped charge- $4 e$ superconductor, as we tune the interaction strength. Numerical simulations of the SU(4) symmetric model [40-42,47-49] point to a single continuous transition, i.e., the SMG transition. In the following, we build a theory for it. One can also think that the charge- $4 e$ interaction $H_{I}$ is related to an interlayer spin-spin interaction as motivated in the Introduction by a suitable particle-hole transformation of two of the four fermion components. The only modification is that we need to consider only the $X Y$ components of the interlayer spin interaction. There are several other choices of interactions $[19,20,35,59]$ that also drive the SMG transition, but for this work, we focus on only the charge- $4 e$ interaction $H_{I}$ described in Eq. (2).

\section{SYMMETRIES}

Symmetries of the model include not only the SU(4) internal symmetry but also the lattice symmetry and the particle-hole symmetry which fixes half filling. The lattice symmetry $G_{\text {latt }}$ includes translation, rotation, and reflection symmetries of the honeycomb lattice. The particle-hole symmetry $\mathbb{Z}_{2}^{\mathcal{S}}$ acts as $\mathcal{S}: c_{i} \rightarrow(-)^{i} c_{i}^{\dagger}$ followed by complex conjugation, such that $\mathcal{S}^{2}=+1$, which is also known as the chiral symmetry or the CT symmetry [60-62]. The combined symmetry $G_{\text {latt }} \times \mathrm{SU}(4) \rtimes \mathbb{Z}_{2}^{\mathcal{S}}$ protects the Dirac semimetal from all fermion bilinear masses and the chemical potential shift. This can be seen from the field theory description for the Dirac semimetal $\mathcal{L}=\sum_{Q=K, K^{\prime}} \bar{c}_{Q} \gamma^{\mu} i \partial_{\mu} c_{Q}$, where $c_{Q}$ is a $\mathrm{SU}(4)$ fundamental spinor at each valley $\left(Q=K, K^{\prime}\right)$. The $\mathrm{SU}(4)$ symmetric bilinear mass terms must take the form of $\bar{c}_{Q} M_{Q Q^{\prime}} c_{Q^{\prime}}$ with a Hermitian $2 \times 2$ matrix $M$ in the valley sector. The space of $M$ is spanned by four Pauli matrices (including $\sigma^{0}$ ) basis, which correspond respectively to the Chern insulator gap, the charge density wave gap, and two Kekule dimerization gaps. The first two break the particlehole and the reflection symmetries and the last two break the translation symmetry, so none of them are allowed by the full symmetry. So the remaining option to generate fermion masses without breaking any symmetry is to invoke fermion interactions, such as the charge- $4 e$ interaction $H_{I}$.

\section{FEATURELESS GAPPED PHASE}

To understand the SMG transition, we need to first understand both sides of the transition. The Dirac semimetal phase is relatively simple. As the interaction is weak and irrelevant, the semimetal phase is well described by the fermion band theory. The featureless gapped phase (the charge-4e superconductor) is more exotic. As the gap is of the many-body nature, it cannot be described by the simple 
band theory picture. Nevertheless, much understanding of the charge- $4 e$ superconductor was obtained by disordering the charge-2e superconductor in previous studies [53-56]. We take the same approach here. Let us consider fermion mass generation in two steps: we first gap the fermion by introducing the charge- $2 e$ pairing at the price of breaking the symmetry, and then we restore the symmetry by disordering the pairing field. The discussion leads to a parton construction for the featureless gapped phase, based on which we can further explore the possibility to merge the two steps of the mass generation into one single transition without the intermediate symmetry breaking phase.

Let us start from the semimetal side and consider the SU(4) sextet pairing on each site (which has six components labeled by $m=1, \ldots, 6)[59,63]$,

$$
\Delta_{i}^{m}=\frac{1}{2} \sum_{a, b} c_{i a} \beta_{a b}^{m} c_{i b},
$$

where $\beta^{m}$ are antisymmetric $4 \times 4$ matrices given by $\boldsymbol{\beta}=\left(\sigma^{12}, \sigma^{20}, \sigma^{32}, i \sigma^{21}, i \sigma^{02}, i \sigma^{23}\right)$, where $\sigma^{\mu \nu}=\sigma^{\mu} \otimes \sigma^{\nu}$ denotes the direct product of Pauli matrices $\sigma^{\mu}$ and $\sigma^{\nu}$. The paring operator $\boldsymbol{\Delta}_{i}$ rotates like an $\mathrm{O}(6)$ vector under the $\mathrm{SU}(4) \cong \operatorname{Spin}(6)$ symmetry, and it transforms under the chiral symmetry as $\mathcal{S}: \boldsymbol{\Delta}_{i} \rightarrow-\boldsymbol{\Delta}_{i}^{\dagger}$. Introducing such a flavor sextet pairing to the Hamiltonian

$$
H_{\boldsymbol{M}}=-\sum_{i} \boldsymbol{M} \cdot\left(\boldsymbol{\Delta}_{i}+\boldsymbol{\Delta}_{i}^{\dagger}\right)
$$

will break the $\mathrm{SU}(4)$ symmetry [down to its $\mathrm{Sp}(2) \cong$ $\operatorname{Spin}(5)$ subgroup] as well as the chiral symmetry $\mathbb{Z}_{2}^{\mathcal{S}}$, and at the same time gap out all the Dirac fermions. In the limit that the paring gap $|\boldsymbol{M}| \rightarrow \infty$, the fermion correlation length shrinks to zero, and the ground state wave function (of $H_{M}$ ) reads

$$
\left|\Psi_{c, \boldsymbol{M}}\right\rangle=\prod_{i}\left(1+\hat{\boldsymbol{M}} \cdot \boldsymbol{\Delta}_{i}^{\dagger}+c_{i 1}^{\dagger} c_{i 2}^{\dagger} c_{i 3}^{\dagger} c_{i 4}^{\dagger}\right)\left|0_{c}\right\rangle,
$$

where $\hat{\boldsymbol{M}}=\boldsymbol{M} /|\boldsymbol{M}|$ is the unit vector that points out the "direction" of the sextet pairing. Comparing $\left|\Psi_{c, \boldsymbol{M}}\right\rangle$ with the wave function $\left|\Psi_{c}\right\rangle$ for the featureless gapped phase in Eq. (3), we can see that the most essential difference lies in the additional fermion bilinear term $\hat{\boldsymbol{M}} \cdot \boldsymbol{\Delta}_{i}^{\dagger}$ in $\left|\Psi_{c, \boldsymbol{M}}\right\rangle$, which breaks the SU(4) symmetry.

To restore the SU(4) symmetry, we need to remove the fermion bilinear term from the wave function. This amounts to symmetrizing the wave function $\left|\Psi_{c, M}\right\rangle$ over all directions of $\boldsymbol{M}$, or in other words, projecting the wave function $\left|\Psi_{c, \boldsymbol{M}}\right\rangle$ to the SU(4) symmetric subspace. Loosely speaking, we propose the following projective construction:

$$
\left|\Psi_{c}\right\rangle \sim \int_{S^{5}} d \boldsymbol{M}\left|\Psi_{c, \boldsymbol{M}}\right\rangle
$$

This construction will be made precise using the parton formalism shortly. But the lesson we learn is that the fermion bilinear mass $\boldsymbol{M}$ serves as a convenient scaffold to construct the featureless gapped state, which can be removed by the symmetrization in the end.

\section{PARTON CONSTRUCTION}

The idea of carrying out the symmetrization on every site invites us to think about "gauging" the SU(4) symmetry as follows. Consider decomposing the physical fermion $c_{i a}$ into bosonic $B_{i a b}$ and fermionic $f_{i b}$ partons $(a, b=1,2,3,4)$,

$$
c_{i a}=\sum_{b=1}^{4} B_{i a b} f_{i b},
$$

with the "orthogonal constraint" [64] on the bosonic parton Hilbert space $\forall a \neq b: \sum_{c} B_{i c a}^{\dagger} B_{i c b}=\sum_{c} B_{i a c} B_{i b c}^{\dagger}=0$. An SU(4) gauge freedom emerges from the fractionalization [65]. On each site, the gauge transformation $U_{i} \in \mathrm{SU}(4)$ is implemented as

$$
B_{i a b} \rightarrow \sum_{c=1}^{4} B_{i a c} U_{i b c}^{*}, \quad f_{i a} \rightarrow \sum_{b=1}^{4} U_{i a b} f_{i b} .
$$

Both the bosonic and the fermionic partons carry the SU(4) gauge charge. Besides the gauge charge, the SU(4) symmetry charge is carried solely by the bosonic parton. The chiral symmetry $\mathbb{Z}_{2}^{\mathcal{S}}$ acts projectively on the partons as $\mathcal{S}: B_{i} \rightarrow-i B_{i}^{\dagger}, f_{i} \rightarrow i(-)^{i} f_{i}^{\dagger}$, where the factor $\pm i$ should be understood as the gauge transform in the $\mathbb{Z}_{4}$ center of the $\mathrm{SU}(4)$ gauge group. So we have $\mathcal{S}^{2}=-1$ for both bosonic and fermionic partons, in contrast to $\mathcal{S}^{2}=+1$ for the physical fermion. As we show later, such a projective $\mathbb{Z}_{2}^{\mathcal{S}}$ action is required by the nontrivial projective symmetry group (PSG) of the parton mean-field theory.

\section{WAVE FUNCTION FROM PARTONS}

Motivated by the previous projective construction, we put the fermionic parton in an SU(4) gauge sextet superconducting state [66] in analogy to Eq. (6),

$$
\left|\Psi_{f}\right\rangle=\prod_{i}\left(1+\hat{\boldsymbol{M}} \cdot \Delta_{i}^{\dagger}[f]+f_{i 1}^{\dagger} f_{i 2}^{\dagger} f_{i 3}^{\dagger} f_{i 4}^{\dagger}\right)\left|0_{f}\right\rangle,
$$

where $\left|0_{f}\right\rangle$ denotes the fermionic parton vacuum state and $\boldsymbol{\Delta}_{i}[f]=\frac{1}{2} \sum_{a, b} f_{i a} \boldsymbol{\beta}_{a b} f_{i b}$ is the gauge sextet paring operator of the fermionic parton $f_{i a}$, which is similar to the flavor sextet pairing of the physical fermion in Eq. (4). With this gauge sextet pairing, the $\mathrm{SU}(4) \cong \operatorname{Spin}(6)$ gauge group is broken down to its $\operatorname{Sp}(2) \cong \operatorname{Spin}(5)$ subgroup. However, the SU(4) symmetry remains untouched, because the symmetry charge is now carried by the bosonic parton. More importantly, the parton state $\left|\Psi_{f}\right\rangle$ is also symmetric 
under the chiral symmetry $\mathbb{Z}_{2}^{\mathcal{S}}$ in the PSG sense [67], which is in contrast to the physical fermion state $\left|\Psi_{c, M}\right\rangle$ in Eq. (6) where $\mathbb{Z}_{2}^{\mathcal{S}}$ is broken. Using the previously proposed PSG transformation $\mathcal{S}: f_{i} \rightarrow i(-)^{i} f_{i}^{\dagger}$, it can be shown that the parton pairing operator transforms as $\mathcal{S}: \boldsymbol{\Delta}_{i}[f] \leftrightarrow \boldsymbol{\Delta}_{i}^{\dagger}[f]$. Hence, the gauge sextet pairing term $\boldsymbol{M} \cdot\left(\boldsymbol{\Delta}_{i}[f]+\boldsymbol{\Delta}_{i}^{\dagger}[f]\right)$ is $\mathbb{Z}_{2}^{\mathcal{S}}$ symmetric, and the resulting mean-field state in Eq. (10) is also symmetric. To construct an SU(4) symmetric state, we consider putting the bosonic parton in a short-range correlated SU(4) singlet state. In the extreme limit of zero correlation length, an SU(4) symmetric many-body state takes the following form:

$$
\left|\Psi_{B}\right\rangle=\prod_{i}\left(1+\frac{1}{4 !} \epsilon_{a b c d} B_{i a 1}^{\dagger} B_{i b 2}^{\dagger} B_{i c 3}^{\dagger} B_{i d 4}^{\dagger}\right)\left|0_{B}\right\rangle,
$$

where $\left|0_{B}\right\rangle$ denotes the bosonic parton vacuum state. $\epsilon_{a b c d}$ is the antisymmetric (Levi-Civita) tensor of four indices, such that the flavor indices are antisymmetrized to form the SU(4) singlet.

Now we take both the bosonic and the fermionic parton wave functions and project them to the physical fermion Hilbert space,

$$
\left|\Psi_{c}\right\rangle=\mathcal{P}\left|\Psi_{B} \Psi_{f}\right\rangle
$$

where the projection operator maps each parton Fock state to the corresponding Fock state of physical fermions:

$$
\mathcal{P}=\prod_{i, a}\left(\left|0_{c}\right\rangle\left\langle 0_{f} 0_{B}\left|+c_{i a}^{\dagger}\right| 0_{c}\right\rangle\left\langle 0_{f} 0_{B}\right| \sum_{b=1}^{4} B_{i a b} f_{i b}\right) .
$$

The resulting state $\left|\Psi_{c}\right\rangle$ in Eq. (12) is precisely the featureless gapped state in Eq. (3). This parton construction provides us with one plausible picture of the featureless gapped phase: the fermionic parton is in a gauge sextet paired state, while the bosonic parton is in an SU(4) symmetric gapped state, and the remaining gauge degrees of freedom are confined. On the other hand, the Dirac semimetal phase also admits a simple picture in the parton formalism: if we put the fermionic parton in the same Dirac band structure as the physical fermion and condense the bosonic parton to the state $\left\langle B_{i a b}\right\rangle=Z \delta_{a b}$ (with $Z$ acting like the quasiparticle weight), then the physical fermion will be identified to the fermionic parton $c_{i a}=Z f_{i a}$ and retrieve the Dirac band structure. We implement these insights in a field theory below.

\section{FIELD THEORY}

What we learned from the parton construction is that the Dirac semimetal and the symmetric massive phase correspond, respectively, to the Higgs and the confined phases of an SU(4) gauge theory. Thus, if there is a direct continuous transition between them, it is conceivable that the transition should be a deconfined critical point [50-52]; i.e., the gauge theory will be deconfined at and only at the transition point. Therefore, we propose the following field theory description for the symmetric mass generation:

$$
\begin{aligned}
\mathcal{L}= & \mathcal{L}_{f}+\mathcal{L}_{B}, \\
\mathcal{L}_{f}= & \sum_{Q=K, K^{\prime}} \bar{f}_{Q} \gamma^{\mu}\left(i \partial_{\mu}-a_{\mu}^{m} \tau^{m}\right) f_{Q}+\mathcal{L}_{\text {int }}, \\
\mathcal{L}_{B}= & -\operatorname{Tr} B\left(i \partial_{\mu}-a_{\mu}^{m} \tau^{m}\right)^{2} B^{\dagger}+r \operatorname{Tr} B B^{\dagger} \\
& +u_{1}\left(\operatorname{Tr} B B^{\dagger}\right)^{2}+u_{2} \operatorname{Tr}\left(B B^{\dagger}\right)^{2} \\
& +u_{3}(\operatorname{det} B+\text { H.c. })+\cdots,
\end{aligned}
$$

which contains the matter fields of bosonic partons $B$ and fermionic partons $f_{Q}$ as well as the SU(4) gauge field $a_{\mu}^{m} \tau^{m}$. The matrices $\tau^{m}(m=1, \ldots, 15)$ are $\mathrm{SU}(4)$ generators (as $4 \times 4$ Hermitian traceless matrices), and $\left(\gamma^{0}, \gamma^{1}, \gamma^{2}\right)=\left(\sigma^{2}, \sigma^{1}, \sigma^{3}\right) . \mathcal{L}_{\text {int }}$ contains short-range interactions of the fermionic parton which we specify later in Eq. (15). This interaction term is treated perturbatively, but it will play an important role to deform the fermionic sector from a pure quantum chromodynamics (QCD) theory, giving rise to possible instabilities of spontaneous mass generation for the fermionic parton in the symmetric gapped phase (as to be analyzed soon). Furthermore, the emergent $\mathrm{U}(1)$ symmetry corresponding to rotating the overall phase of the fermionic parton $\left(f_{Q} \rightarrow e^{i \theta} f_{Q}\right)$ will also be broken by the interaction $\mathcal{L}_{\text {int }}$.

To reformulate the fractionalization scheme Eq. (8) at the field theory level, we start with the low-energy physical fermions $c_{Q}\left(Q=K, K^{\prime}\right)$ around $K$ and $K^{\prime}$ points of the Brillouin zone. Both of them transform under the SU(4) symmetry as fundamental representations. We can fractionalize the physical fermion field to the parton fields as $c_{Q}=B \cdot f_{Q}$, where $f_{Q}$ is a four component fermion field [transforming as a SU(4) gauge fundamental] for each valley $Q$, and $B$ is a $4 \times 4$ matrix field that transforms under both the SU(4) symmetry (from left) and the SU(4) gauge symmetry (from right). Based on the fractionalization scheme of Eq. (8), we expect the matrix field $B$ to be unitary (up to normalization constant $Z$ ) on the lattice scale. The constraint may be imposed by a Lagrangian multiplier $\lambda \operatorname{Tr}\left(B B^{\dagger}-Z^{2}\right)^{2}$, which, under renormalization, leads to an effective potential for $B$ in the field theory, whose leading terms ( $r$ and $u_{1,2}$ terms of $\mathcal{L}_{B}$ ) are given in Eq. (14). The $u_{3}$ term is another $\mathrm{SU}(4)$ symmetric four-boson interaction, which explicitly breaks the U(1) symmetry of $B$ and can be viewed as a descendant of the charge- $4 e$ superconducting interaction $H_{I}$ in Eq. (2).

\section{SYMMETRIC GAPPED PHASE}

In the field theory Eq. (14), the SMG transition is driven by $r$. When $r>0$, the bosonic parton is gapped, leaving the 
fermionic parton coupled to the SU(4) gauge field below the scale of the bosonic parton gap $\Delta_{B}$, described by the $N_{f}=2 \mathrm{SU}(4)$ QCD theory. We assume that this $\mathrm{SU}(4)$ QCD theory is confining [68]. The resulting confined phase will depend on additional details. For example, if we considered a pure SU(4) QCD, with no additional fourfermion interactions, a $\mathrm{U}(1)$ symmetry of $f_{Q} \rightarrow e^{i \theta} f_{Q}$ (the baryon number conservation) will be present, and will not be broken in the confined phase, by the Vafa-Witten theorem [69]. Instead, chiral symmetry breaking is likely to occur, breaking the $\mathrm{SU}(2)$ valley symmetry. However, for our purposes it is crucial to include the following fourfermion interaction term in the form of the pair-pair interaction of the gauge sextet pairing $\boldsymbol{\Delta}[f]=f_{K}^{\mathrm{T}} i \gamma^{0} \boldsymbol{\beta} f_{K^{\prime}}$ :

$$
\mathcal{L}_{\text {int }}=\frac{g}{2}(\boldsymbol{\Delta}[f] \cdot \boldsymbol{\Delta}[f]+\text { H.c. }) .
$$

This interaction can be written as $f_{1} f_{2} f_{3} f_{4}+$ H.c. equivalently [which is SU(4) gauge neutral the same as $\boldsymbol{\Delta} \cdot \boldsymbol{\Delta}$ ], reminiscent of the charge- $4 e$ interaction $V$ between electrons in Eq. (2) that drives the transition. Now, the U(1) baryon number is no longer a global symmetry of the theory and Vafa-Witten does not forbid mass generation in the U(1) breaking (gauge sextet pairing) channel.

If the SU(4) gauge fluctuation were absent, the shortrange interaction $\mathcal{L}_{\text {int }}$ would be perturbatively irrelevant, given the negative engineering dimension $[g]=-1<0$ of the coupling $g$. As the $\mathrm{SU}(4)$ gauge fluctuation is included in the QCD theory, the scaling dimension $[g]$ can receive anomalous dimension corrections. By a controlled renormalization group (RG) analysis based on the $1 / N_{f}$ expansion, detailed in Appendix A, we compute the scaling dimension $[g]=-1+80 /\left(\pi^{2} N_{f}\right)$ to the $1 / N_{f}$ order, implying that the interaction $\mathcal{L}_{\text {int }}$ could become relevant (i.e., $[g]>0$ ) as we push $N_{f}$ to $N_{f}=2$. As the interaction flows strong under $\mathrm{RG}$, it will drive the condensation of the gauge sextet pairing and lead to a mass term $\boldsymbol{M} \cdot(\boldsymbol{\Delta}[f]+$ H.c. $)$, which will gap the fermionic partons, at the scale of $\Delta_{f} \sim|\boldsymbol{M}|$, and break the gauge group down to $\mathrm{Sp}(2)$. In the absence of matter field fluctuations below the energy scale $\Delta_{f}$, the non-Abelian $\mathrm{Sp}(2)$ gauge field will confine itself (at a confinement scale $\Delta_{a}$ ). However, the SU(4) symmetry remains unbroken, since the bosonic parton is gapped and disordered. The particle-hole symmetry $\mathcal{S}$ also remains unbroken because it is realized on the fermionic partons projectively, $\mathcal{S}: f_{Q} \rightarrow i f_{Q}^{\dagger}$, where the $\mathbb{Z}_{4}$ gauge transformation is crucial to undo the sign change of $\boldsymbol{M}$ originally caused by the particle-hole transformation. Thus, the system is in the featureless gapped phase that preserves all symmetries.

\section{MASSLESS DIRAC PHASE}

When $r<0$, the bosonic parton condenses $\langle B\rangle \neq 0$. A positive $u_{2}>0$ term would favor the condensate configuration $\langle B\rangle$ to be a unitary matrix (up to an overall factor $Z$ ). Thus, we can always choose $\left\langle B_{a b}\right\rangle=Z \delta_{a b}$ by $\mathrm{SU}(4)$ gauge transformations. This will identify the SU(4) symmetry with the SU(4) gauge group and Higgs out all gauge fluctuations. The system is then in the Dirac semimetal phase, described by $\mathcal{L}=\sum_{Q=K, K^{\prime}} \bar{c}_{Q} \gamma^{\mu} i \partial_{\mu} c_{Q}$, where $c_{Q}=Z f_{Q}$ and $Z$ may be interpreted as the quasiparticle weight. Any short-range interaction $\mathcal{L}_{\text {int }}$ among the fermionic parton in Eq. (14) will become perturbatively irrelevant once the SU(4) gauge fluctuation is Higgs out by the condensation of the bosonic parton, and the corresponding interaction-driven instability (such as the gauge sextet pairing instability) will cease to exist. In this way, the $\mathrm{RG}$ relevance of the fermionic parton interaction $\mathcal{L}_{\text {int }}$ (and the fermionic parton mass generation) is controlled by the mass $r$ of the bosonic parton, so the SMG does not need fine-tuning (other than the only driving parameter $r$ ).

\section{CRITICAL POINT}

At $r=0$, both fermionic $\left(f_{Q}\right)$ and bosonic $(B)$ partons are gapless. Together, they screen the SU(4) gauge field more efficiently, hence reducing the tendency to confinement. This opens up the possibility of a stable deconfined SU(4) QCD-Higgs theory, which could describe the SMG critical point. We note that the similar behavior, namely, the gauge confinement being irrelevant [52] at the critical point due to the gapless bosons, was also discussed in the deconfined phase transition [50,51] between the Néel and the valence bond solid phases. On moving away from the critical point into the phase where the Higgs fields are gapped, confinement takes over.

Based on the above understanding, the energy scales $\Delta_{B}$, $\Delta_{f}$, and $\Delta_{a}$ should catch up one after another as we enter the featureless gapped phase, as illustrated in Fig. 2(a). This implies a hierarchy of length scales $\xi_{B, f, a} \sim \Delta_{B, f, a}^{-1}$ near the SMG transition from the side of the featureless gapped phase as shown in Fig. 2(b). For example, the SU(4) multiplet fluctuation will be gapped with the bosonic parton at the length scale of $\xi_{B}$. However, the SU(4) singlet fluctuation can persist to a longer length scale $\xi_{a}$ until the $\mathrm{Sp}(2)$ gluon gets confined.
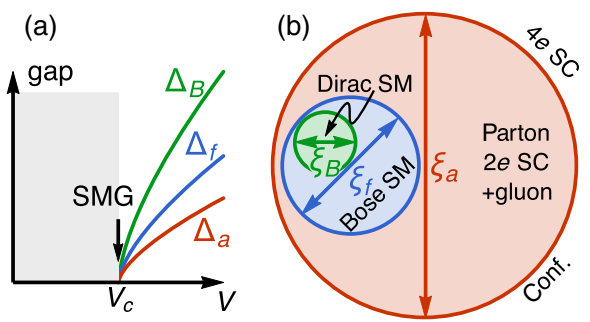

FIG. 2. (a) Catching-up energy scales of the bosonic parton gap $\Delta_{B}$, the fermionic parton gap $\Delta_{f}$, and the gauge gluon gap $\Delta_{a}$ on the confinement side of the SMG. (b) Hierarchical length scales of $\xi_{B}, \xi_{f}$, and $\xi_{a}$ near the SMG transition. 
The above field theory description is in parallel with the parton construction as discussed previously. But the field theory also provides other possible scenarios for the transition(s) between the Dirac semimetal and the symmetric gapped phase, when the gaps $\Delta_{B}, \Delta_{f}, \Delta_{a}$ fail to open up together as the interaction $V / t$ increases. For example, by tuning the short-range interactions of the fermionic parton $f_{Q}$ in Eq. (14), it is possible that the fermionic parton may develop the bilinear mass before the bosonic parton is gapped, an intermediate SU(4) symmetry breaking charge- $2 e$ superconducting phase will set in, with the condensation of the SU(4) sextet Cooper pairs of the physical fermion, as shown in Fig. 1(a). Such a charge$2 e$ superconducting phase was also observed in numerical simulations if the lattice model in Eqs. (1) and (2) is deformed by the attractive Hubbard interaction [70-72] or by doping the chemical potential away from the Dirac point [58].

A more exotic scenario occurs if an extended deconfined phase is present, leading to an intermediate gapless quantum liquid, that is, if the fermion parton mass generation and the gauge confinement happens after the gapping of the bosonic parton, as shown in Fig. 1(c). In this phase, the physical fermions are gapped, and the lowenergy bosonic fluctuations are described by a wave function obtained from the gauge projection of the fermionic parton semimetal state. Therefore, we may call it a Bose semimetal (BSM) phase [43-46]. The gapless bosonic fluctuation should be $\mathrm{SU}(4)$ singlets and transform only under the lattice symmetry. A possible candidate is the valence bond solid order fluctuation. Dynamically, which of these scenarios are more favorable should depend on the details of parton interactions and gauge dynamics. Numerical evidence from the lattice model seems to support a direct continuous transition without either of the intermediate phases, as shown in Fig. 1(b).

\section{FERMION GREEN'S FUNCTION}

One smoking-gun "feature" of the featureless gapped phase is the existence of zeros in the fermion Green's function at zero frequency [73]. To be precise, let us define the Green's function of the physical fermion to be $G_{a b}(x)=-\left\langle c_{a}(x) \bar{c}_{b}(0)\right\rangle$, where $x=(t, \boldsymbol{x})$ is the spacetime coordinate. Fourier transforming to the momentumfrequency space, we have $G(k)=\int d^{3} x G(x) e^{i k_{\mu} x^{\mu}}$, with $k=(\omega, \boldsymbol{k})$. In the Dirac semimetal phase, poles of the Green's function appear along the band dispersion. In particular, at the $K$ and $K^{\prime}$ points of the Brillouin zone where the fermion becomes gapless, the pole is pushed to zero frequency, and, hence, $G(k) \sim \omega^{-1}$. However, in the symmetric gapped phase, as proven in Ref. [73], the poles will be replaced by zeros: $G(k) \sim \omega$ as $\omega \rightarrow 0$ at $\boldsymbol{k}=K, K^{\prime}$. In fact, the Green's function zeros are symmetry protected in the featureless gapped phase, which was proved in Ref. [73]. Let us focus in the vicinity of the $K$ and $K^{\prime}$ points

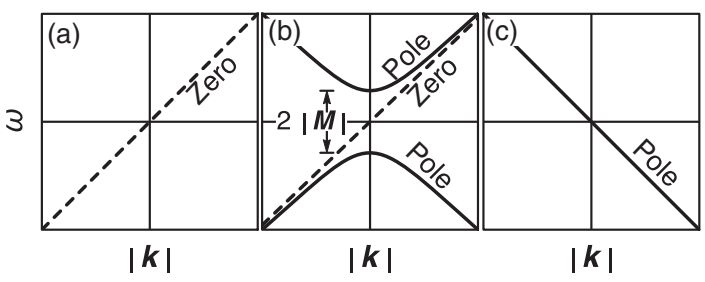

FIG. 3. The zero-pole transition of the fermion Green's function. Take the one of the $G(k)$ eigenvalues $(\omega-|\boldsymbol{k}|) /\left(\omega^{2}-\right.$ $|\boldsymbol{k}|^{2}-|\boldsymbol{M}|^{2}$ ), for example.

and redefine $\boldsymbol{k}$ to be the momentum deviation from them. The SMG transition is also a zero-pole transition in the fermion Green's function at $k \rightarrow 0$. Let us see how this is reproduced by the parton theory.

Using the parton construction outlined in Eq. (7), we can calculate the fermion Green's function deep in the featureless gapped phase. The result is (see Appendix B for details)

$$
G_{a b}(k)=\frac{\gamma^{\mu} k_{\mu}}{k^{\mu} k_{\mu}+M^{2}} \delta_{a b},
$$

where $|\boldsymbol{M}|$ corresponds to the sextet pairing gap of the fermionic parton. In the featureless gapped phase (where $|\boldsymbol{M}|$ is finite), $G(k)$ approaches zero analytically at $k \rightarrow 0$ as expected; see Fig. 3(a). This lends confidence to our parton construction of the featureless gapped phase. The fact that the quasiparticle weight approaches unity deep in the featureless gapped phase is also consistent with expectation. Because the charge- $4 e$ superconducting ground state is a fully gapped symmetric short-range entangled state (similar to a vacuum state), a physical fermion $c$ doped into the system will just propagate as a quasiparticle above its spectral gap set by the mass scale $|\boldsymbol{M}|$ without any fractionalization [which is also consistent with the picture that the SU(4) gauge theory is confining in the featureless gapped phase].

The Green's function $G(k)$ in Eq. (16) also provides a plausible scenario for the zero-pole transition. As the gap $|\boldsymbol{M}|$ decreases, two branches of poles are brought down from high energy, as shown in Fig. 3(b). They approach the line of zero asymptotically and eventually annihilate with the zero at the SMG transition where $|\boldsymbol{M}| \rightarrow 0$. Then only a line of pole is left in the Dirac semimetal phase in Fig. 3(c). A similar mechanism for the zero-pole transition was proposed in Ref. [74]. However, we also note that the Green's function $G(k)$ in Eq. (16) cannot describe the fermion correlation close to the SMG critical point, where the bosonic parton and the gauge fluctuation also become important, such that one needs to go beyond the variational approach to describe the vanishing quasiparticle weight and the continuum spectral function as a result of the fermion fractionalization.

\section{CONCLUSION AND DISCUSSION}

The SMG is an exotic quantum phase transition between the Dirac semimetal phase and a symmetric gapped phase, 
which cannot be understood within conventional theories of Dirac mass generation such as the Gross-Neveau mechanism. However, it has been numerically observed in different models with different interaction and symmetries. We propose a theoretical framework for SMG broadly as a deconfined quantum critical point, where the physical fermion is fractionalized into bosonic and fermionic partons with emergent gauge interaction. The Dirac semimetal phase corresponds to the Higgs phase, and the featureless gapped phase corresponds to the confined phase. The gauge group, the parton flavor number, and the symmetry assignment are flexible components of the theoretical framework that can be adapted to the model details, including the interaction parameters and the model symmetries. More work is required to understand what determines these parameters.

In this work, we propose that SMG in $(2+1) \mathrm{D}$ with SU(4) global symmetry can be described by an SU(4) $\mathrm{QCD}_{3}$-Higgs theory. Analyzing the nonperturbative dynamics of such a strongly coupled critical point is currently beyond our analytical capabilities, but a few statements can be made based on the basic structure of the theory following the approach used in $\mathrm{U}(1)$ deconfined quantum criticality $[75,76]$. At the critical point we expect an emergent $\mathrm{SU}(2)$ symmetry in the valley space, relating a pair of valence bond solid orders and staggered A/B sublattice order on the honeycomb lattice. Potentially, there is an additional charge $\mathrm{U}(1)$ symmetry that could emerge at the critical point if either the $u_{3} \operatorname{Re} \operatorname{det}(B)$ in the bosonic parton sector or $\mathcal{L}_{\text {int }}$ of the fermionic partons is irrelevant at the transition. However, this appears unlikely since the term that drives the transition, the four-electron charge- $4 e$ superconductor term, itself breaks this symmetry. It will be interesting to test the enlarged symmetry of the SMG critical point in numerics. Another prediction is that the anomalous dimension of the electrons is large at criticality since it decays into a pair of partons. Similarly, the SU(4) symmetry order parameters which are bilinears in the $B$ field should also have large anomalous dimension. For example, the quantum Monte Carlo simulation in Ref. [40] obtained the anomalous dimension $\eta_{\mathrm{SMG}}=0.7 \pm 0.1$ for the $\mathrm{O}(6)$ order parameter, much larger than $\eta_{\mathrm{WF}}=0.035$ at the $\mathrm{O}(6)$ Wilson-Fisher fixed point.

The theoretical framework proposed in this work may be applied to the SMG with other symmetry groups and in other dimensions. For example, in an upcoming work [77], we will study SMG in a model with lower symmetry, $\mathrm{SU}(2) \times \mathrm{SU}(2) \times \mathrm{SU}(2)$, which will be described by a $\mathrm{SU}(2) \mathrm{QCD}_{3}$-Higgs theory. The advantage of the lower symmetry model is that it will give us access to more phases, and we can check if our critical theory can be perturbed to obtain the larger phase diagram. The largest symmetry group for $(2+1) \mathrm{D}$ SMG is $\mathrm{SO}(7)$, where we can still consider a honeycomb lattice model with eight Majorana fermions on each site, transforming like a SO(7) spinor. The $\mathrm{SO}(7) \mathrm{SMG}$ can be driven by applying the SO(7) symmetric Fidkowski-Kitaev interaction $[19,20]$ to each site. All the lower symmetry SMGs in 2D can be considered as descendants of the $\mathrm{SO}$ (7) SMG by partially breaking the $\mathrm{SO}(7)$ symmetry down to its subgroup. An interesting direction is to consider SMG in various dimensions. In $(3+1) \mathrm{D}$, once again it is readily shown that eight Dirac fermions (or 16 Weyl fermions) can be gapped to produce a featureless state [39,59,78-81]. Whether this can proceed through a single continuous transition remains to be seen: numerics on one microscopic model appear to give an intervening symmetry breaking phase [49]. One may also discuss SMG in $(1+1) \mathrm{D}$, where we need four Dirac fermions. In fact, this is closely related to the interaction reduction of topological phases in $(1+1) \mathrm{D}$ described by FidkowskiKitaev [19], where they show that edge states with eight Majorana modes are unstable despite the presence of timereversal symmetry that forbids a quadratic gapping term. Therefore, the transition between the trivial phase and a phase with eight Majorana (or four Dirac) edge zero modes can be circumvented by interactions, which is related to symmetric mass generation for four Dirac fermions in $(1+1) D$. In Appendix $C$, we review and reinterpret the Fidkowski-Kitaev transition in $(1+1) \mathrm{D}$ within an SO(7) SMG [19] in the parton language. In Appendix $\mathrm{C}$, we provide an alternative formulation of the problem by a set of partons similar to our previous construction, and also informed by the $\mathrm{SO}(8)$ triality, within which the transition is simply described. Could such a change of variable or duality transformation be constructed to describe SMG in higher dimensions? These are questions for future work.

\section{ACKNOWLEDGMENTS}

We would like to acknowledge the helpful discussions with Max Metlitski, N. Seiberg, T. Senthil, Chong Wang, Andreas Ludwig, John McGreevy, Tarun Grover, Xiao-Liang Qi, Yingfei Gu, Aneesh Manohar, Subir Sachdev, and Leon Balents. A. V. and Y.-Z. Y. were supported by a Simons Investigator grant. Y.-C. H. is supported by a postdoctoral fellowship from the Gordon and Betty Moore Foundation, under the EPiQS initiative, GBMF4306, at Harvard University. C.X. is supported by the David and Lucile Packard Foundation and NSF Grant No. DMR-1151208.

\section{APPENDIX A: RENORMALIZATION GROUP ANALYSIS}

In this appendix, we present the renormalization group (RG) analysis of the $N_{f}=2 \mathrm{SU}(4)$ QCD theory with shortrange fermion interaction. The theory arises from the SMG field theory Eq. (14) after gapping out the bosonic field $B$. To control the RG calculation, we can generalize the theory to the large- $N_{f}$ limit. The Lagrangian in consideration reads 


$$
\mathcal{L}_{f}=\sum_{a=1}^{N_{f}} \sum_{i, j} \sum_{\alpha, \beta} \bar{f}_{a \alpha i} \gamma_{\alpha \beta}^{\mu}\left(i \partial_{\mu}-a_{\mu}^{m} \tau_{i j}^{m}\right) f_{a \beta j}+\mathcal{L}_{\text {int }} .
$$

The fermionic parton field $f_{a \alpha i}$ is labeled by the flavor index $a=1, \ldots, N_{f}$, the Dirac index $\alpha=1,2$, and the color index $i=1,2,3,4$. The flavor indices are transformed under the flavor symmetry group $\operatorname{Sp}\left(N_{f} / 2\right)$ and the color indices are transformed under the gauge group SU(4). The case of $N_{f}=2$ is relevant to our discussion in the main text. $a_{\mu}^{m}$ is the SU(4) gauge field that couples to the fermion via the SU(4) generators $\tau^{m}$ acting in the color subspace. The SU(2) rotation in the Dirac subspace corresponds to the spacetime rotation. The $\gamma^{\mu}$ matrices are chosen as $\left(\gamma^{0}, \gamma^{1}, \gamma^{2}\right)=$ $\left(\sigma^{2}, \sigma^{1}, \sigma^{3}\right)$ and $\bar{f}_{a \alpha i}=f_{a \beta i}^{\dagger} \gamma_{\beta \alpha}^{0}$.

$\mathcal{L}_{\text {int }}$ denotes the short-range four-fermion interaction. The charge- $4 e$ superconducting interaction for the physical fermion will naturally induce a similar interaction for the fermionic parton with the same symmetry properties. It can be verified that the following interaction is the only fourfermion interaction that is invariant under the spacetime rotation, the $\mathrm{Sp}\left(N_{f} / 2\right)$ symmetry, and the $\mathrm{SU}(4)$ gauge transformations, but breaks the U(1) symmetry in the same manner as the physical fermion interaction.

$$
\begin{aligned}
\mathcal{L}_{\text {int }}= & g\left(V_{\alpha \beta \gamma \delta}^{a b c d} \epsilon_{i j k l} f_{a \alpha i} f_{b \beta j} f_{c \gamma k} f_{d \delta l}+\text { H.c. }\right), \\
V_{\alpha \beta \gamma \delta}^{a b c d}= & J_{a b} J_{c d} \epsilon_{\alpha \beta} \epsilon_{\gamma \delta}+J_{a c} J_{b d} \epsilon_{\alpha \gamma} \epsilon_{\beta \delta} \\
& +J_{a d} J_{b c} \epsilon_{\alpha \delta} \epsilon_{\beta \gamma},
\end{aligned}
$$

where $\epsilon_{i j k l}$ is the totally antisymmetric tensor in the color subspace, $\epsilon_{\alpha \beta}$ is the antisymmetric matrix in the Dirac subspace, and $J_{a b}$ is the symplectic form of the $\operatorname{Sp}\left(N_{f} / 2\right)$ group in the flavor subspace [such that the generator $A$ of $\mathrm{Sp}\left(N_{f} / 2\right)$ preserves $\left.J A+A^{\top} J=0\right]$.

Figure 4 concludes the diagrams that contribute to the linear order (in $g$ ) of the RG equation at the $1 / N_{f}$ order. Following Refs. [82,83], the RG flow equation is given by

$$
\frac{d g}{d \ell}=-\left(1-\frac{80}{\pi^{2} N_{f}}\right) g
$$

So the interaction strength $g$ has the scaling dimension $[g]=-1+80 /\left(\pi^{2} N_{f}\right)+\mathcal{O}\left(N_{f}^{-2}\right)$. At $N_{f}=2$, we have

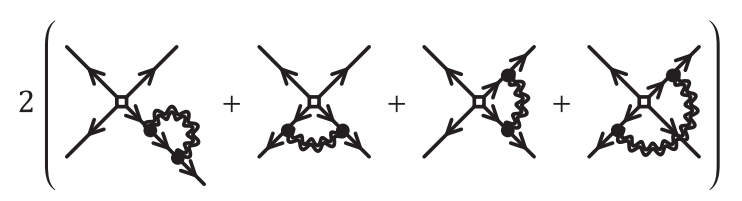

FIG. 4. Diagrams that contributes the correction of the interaction vertex. Wavy lines are the gauge boson propagators $D(q)_{\mu \nu}^{m n}=16 q^{-1}\left(\delta_{\mu \nu}-\xi q_{\mu} q_{\nu} / q^{2}\right) \delta_{m n}$ at the large- $N_{f}$ fixed point. The arrowed lines are the fermion propagator $G(k)=1 /\left(k_{\mu} \gamma^{\mu}\right)$. $[g] \approx 3.05>0$, implying that the short-range interaction $g$ is relevant. To analyze the instabilities due to this interaction, we recall the $\boldsymbol{\beta}=\left(\sigma^{12}, \sigma^{20}, \sigma^{32}, i \sigma^{21}, i \sigma^{02}, i \sigma^{23}\right)$ matrices defined in the main text, then the antisymmetric tensor $\epsilon_{i j k l}$ can be decomposed as $\epsilon_{i j k l}=\frac{1}{2} \sum_{m} \beta_{i j}^{m} \beta_{k l}^{m}$. Thus, at $N_{f}=2$, the interaction in Eq. (A2) becomes

$$
\mathcal{L}_{\text {int }}=\frac{g}{2}\left[\left(f_{K}^{\top} i \gamma^{0} \boldsymbol{\beta} f_{K^{\prime}}\right)^{2}+\text { H.c. }\right] \text {. }
$$

So the strong charge- $4 e$ interaction could drive the spontaneous generation of the gauge sextet pairing $\boldsymbol{\Delta}=f_{K}^{\top} i \gamma^{0} \boldsymbol{\beta} f_{K^{\prime}}$ regardless of the sign of $g$. The sign of $g$ only determines whether the instability is in the $\operatorname{Re} \Delta$ channel $(g<0)$ or in the $\operatorname{Im} \Delta$ channel $(g>0)$. But either case will lead to the fermionic parton mass generation and the gauge confinement, so the sign of $g$ is not important.

\section{APPENDIX B: DERIVATION OF THE GREEN'S FUNCTION}

As we show in Eq. (7), in the extreme limit of zero correlation length, the featureless gapped state $\left|\Psi_{c}\right\rangle$ in Eq. (3) can be obtained exactly by projecting the mean-field state $\left|\Psi_{c, M}\right\rangle$ to the SU(4) symmetric sector. In this appendix, we generalize this construction to the case of finite correlation length. Although the projective construction will not be exact as we go away from the zero correlation length limit, it still provides us a useful variational wave function which has a controlled asymptotically exact limit, based on which we can evaluate the fermion Green's function.

The idea to increase the fermion correlation in the wave function $\left|\Psi_{c}\right\rangle$ is to allow the fermion to move around on the lattice. So we turn on the fermion hopping term in the mean-field Hamiltonian:

$$
H_{\mathrm{MF}}=H_{0}+H_{\boldsymbol{M}}=-t \sum_{\langle i j\rangle} c_{i}^{\dagger} c_{j}-\sum_{i} \boldsymbol{M} \cdot \boldsymbol{\Delta}_{i}+\text { H.c. }
$$

Let us still denote the mean-field ground state as $\left|\Psi_{c, \boldsymbol{M}}\right\rangle$. Switching to the momentum-frequency space and using the Nambu spinor basis $c_{k}=\left(c_{K+k}, c_{K^{\prime}+k}^{\dagger}\right)^{\top}$, the fermion correlation on the mean-field state is given by

$$
\begin{aligned}
G_{\boldsymbol{M}}(k) & =-\left\langle\Psi_{c, \boldsymbol{M}}\left|c_{k} \bar{c}_{k}\right| \Psi_{c, \boldsymbol{M}}\right\rangle \\
& \simeq\left[\begin{array}{cc}
\gamma^{\mu} k_{\mu} & -i \boldsymbol{M} \cdot \boldsymbol{\beta} \\
i \boldsymbol{M} \cdot \boldsymbol{\beta} & -\gamma^{\mu} k_{\mu}
\end{array}\right]^{-1} \\
& =\frac{1}{k^{\mu} k_{\mu}+\boldsymbol{M}^{2}}\left[\begin{array}{cc}
\gamma^{\mu} k_{\mu} & -i \boldsymbol{M} \cdot \boldsymbol{\beta} \\
i \boldsymbol{M} \cdot \boldsymbol{\beta} & -\gamma^{\mu} k_{\mu}
\end{array}\right]
\end{aligned}
$$

at low energy. We propose an SU(4) symmetric wave function $\left|\Psi_{c}\right\rangle$ by symmetrizing $\left|\Psi_{c, M}\right\rangle$ following Eq. (7), as 
$\left|\Psi_{c}\right\rangle=\int_{S^{5}} d \boldsymbol{M}\left|\Psi_{c, \boldsymbol{M}}\right\rangle$ [assuming the measure is $\mathrm{SO}(6)$ symmetric and is properly normalized], where $S^{5}$ denotes a sphere of radius $|\boldsymbol{M}|$. Then the Green's function on the symmetric state can be obtained by symmetrizing the mean-field Green's function. To see this, we start with

$$
\begin{aligned}
G(k) & =-\left\langle\Psi_{c}\left|c_{k} \bar{c}_{k}\right| \Psi_{c}\right\rangle \\
& =-\int d \boldsymbol{M} d \boldsymbol{M}^{\prime}\left\langle\Psi_{c, \boldsymbol{M}}\left|c_{k} \bar{c}_{k}\right| \Psi_{c, \boldsymbol{M}^{\prime}}\right\rangle
\end{aligned}
$$

The overlap $\left\langle\Psi_{c, \boldsymbol{M}}\left|c_{k} \bar{c}_{k}\right| \Psi_{c, \boldsymbol{M}^{\prime}}\right\rangle$ vanishes if $\boldsymbol{M} \neq \boldsymbol{M}^{\prime}$, due to the orthogonality catastrophe of fermion many-body states. Therefore, we have

$$
\begin{aligned}
G(k) & =-\int d \boldsymbol{M}\left\langle\Psi_{c, \boldsymbol{M}}\left|c_{k} \bar{c}_{k}\right| \Psi_{c, \boldsymbol{M}}\right\rangle \\
& =\int d \boldsymbol{M} G_{\boldsymbol{M}}(k) .
\end{aligned}
$$

The symmetrization will remove the $\boldsymbol{M} \cdot \boldsymbol{\beta}$ terms in the numerator but leave the $\boldsymbol{M}^{2}$ term in the denominator untouched. Switching back from the Nambu basis, we arrive at the Green's function in Eq. (16).

\section{APPENDIX C: FIDKOWSKI-KITAEV SO(7) SYMMETRIC MASS GENERATION}

In this appendix, we review the $(1+1) \mathrm{D}$ FidkowskiKitaev SO(7) symmetric mass generation $[19,20]$ from the perspective of the parton construction. The model is defined on a 1D lattice. On each site, there are eight Majorana fermion modes $\chi_{i a}(a=1, \ldots, 8)$ forming the eight-dimensional real spinor representation of an $\mathrm{SO}(7)$ group. The Hamiltonian reads $H=H_{0}+H_{I}$, with

$$
\begin{aligned}
& H_{0}=\sum_{i} \sum_{a=1}^{8} i \chi_{i, a} \chi_{i+1, a}, \\
& H_{I}=-\frac{V}{4 !} \sum_{i} \sum_{m=1}^{7} \Delta_{i}^{m} \Delta_{i}^{m},
\end{aligned}
$$

where $\Delta_{i}^{m}=\chi_{i a} \Gamma_{a b}^{m} \chi_{i b}$ are the seven components of the $\mathrm{SO}(7)$ vector. The Gamma matrices can be chosen as $\boldsymbol{\Gamma}=\left(\sigma^{123}, \sigma^{203}, \sigma^{323}, \sigma^{211}, \sigma^{021}, \sigma^{231}, \sigma^{002}\right)$, which form a set of purely imaginary, antisymmetric, and anticommuting matrices. The Hamiltonian $H$ is manifestly $\mathrm{SO}(7)$ invariant. Besides the internal $\mathrm{SO}(7)$ symmetry, the model also possesses the translation symmetry $T: \chi_{i} \rightarrow \chi_{i+1}$ and the chiral symmetry $\mathcal{S}: \chi_{i} \rightarrow(-)^{i} \chi_{i}, i \rightarrow-i$. One can see $\mathcal{S}^{2}=+1$ and $T^{-1} \mathcal{S} T \mathcal{S}=-1$ acting on the fermions.

In the noninteracting limit $(V \rightarrow 0), H_{0}$ simply describes eight decoupled and gapless Majorana chains, whose field theory description is

$$
\mathcal{L}_{0}=\frac{1}{2} \sum_{a=1}^{8} \bar{\chi}_{a} \gamma^{\mu} i \partial_{\mu} \chi_{a}
$$

where $\chi_{a}=\left(\chi_{L a}, \chi_{R a}\right)^{\top}$ and $\bar{\chi}_{a}=\chi_{a}^{\top} \gamma^{0}$ contain both leftand right-moving Majorana modes with the gamma matrices $\left(\gamma^{0}, \gamma^{1}\right)=\left(i \sigma^{2}, \sigma^{1}\right)$. Since the Majorana coupling along the chain is purely imaginary, the Fermi points are located at momentum $k=0, \pi$, so $\chi_{L, R}$ fields are related to the realspace fermion $\chi_{i}$ by

$$
\chi_{L a}=\sum_{i} \chi_{i a}, \quad \chi_{R a}=\sum_{i}(-)^{i} \chi_{i a} .
$$

Both $\chi_{L}$ and $\chi_{R}$ transform as $\mathrm{SO}(7)$ spinors. The translation and the chiral symmetry act as

$$
T: \chi_{R a} \rightarrow-\chi_{R a}, \quad \mathcal{S}: \chi_{L a} \leftrightarrow \chi_{R a} .
$$

All fermion bilinear mass terms (such as $i m \bar{\chi} \chi$ ) are forbidden by these symmetries. In fact, the translation symmetry is the most important protecting symmetry which is sufficient to rule out all bilinear masses (i.e., all backscattering terms between $\chi_{L}$ and $\chi_{R}$ ). So the Majorana chain cannot be symmetrically gapped on the free-fermion level.

However, it is possible to symmetrically gap out eight Majorana chains by fermion interactions. One possible interaction proposed by Fidkowski and Kitaev $[19,20]$ is the $\mathrm{SO}(7)$ symmetric interaction $V$ in Eq. (C1) (abbreviated as the FK interaction hereafter). In the strong interaction limit $(V \rightarrow+\infty)$, the Hamiltonian is dominated by $H_{I}$, which decouples to each single site. Diagonalizing the onsite Hamiltonian, one finds a unique ground state separated from the excited states by a finite gap $\Delta=14 \mathrm{~V}$. If we pair up the on-site Majorana fermions into Dirac fermions as $c_{i a}=\chi_{i, 2 a-1}+i \chi_{i, 2 a}(a=1, \ldots, 4)$, the ground state wave function can be expressed as

$$
\left|\Psi_{c}\right\rangle=\prod_{i}\left(1+c_{i 1}^{\dagger} c_{i 2}^{\dagger} c_{i 3}^{\dagger} c_{i 4}^{\dagger}\right)\left|0_{c}\right\rangle,
$$

which is the $1 \mathrm{D}$ version of the charge- $4 e$ superconducting state in Eq. (3). It can be verified that $\left|\Psi_{c}\right\rangle$ preserves the full $\mathrm{SO}(7)$, translation, and chiral symmetries, and hence a featureless gapped state. The only difference with the $(2+1) \mathrm{D}$ case is that in $(1+1) \mathrm{D}$ the interaction $V$ is marginal at the free-fermion fixed point. Depending on the sign of $V$, it is marginally relevant if $V>0$ and marginally irrelevant if $V<0$. So as long as we turn on an infinitesimal but positive $V$, the system undergoes a 1D version of the symmetric mass generation to the featureless gapped phase.

A better understanding of this 1D SMG physics would greatly help us to understand the SMG transitions in all higher dimensions. In the following, we present a parton construction following Ref. [19] using the $\mathrm{SO}(8)$ triality. 
The $\mathrm{SO}(8)$ triality is a property that the $\mathrm{SO}(8)$ vector 8 , left-spinor $\mathbf{8}_{+}$, and right-spinor $\mathbf{8}_{-}$representations can fuse to the trivial representation under the trilinear map $t: \mathcal{V}_{\mathbf{8}} \times \mathcal{V}_{\mathbf{8}_{+}} \times \mathcal{V}_{\mathbf{8}_{-}} \rightarrow \mathbb{R}$. The trality map $t$ can also be written as a three-leg tensor $t_{a b}^{m}$, where the tensor indices $m$, $a, b$ label the basis of $\mathbf{8}, \mathbf{8}_{+}$, and $\mathbf{8}_{-}$, respectively. Without interactions, $H_{0}$ has the full $\mathrm{SO}(8)$ symmetry that rotates the eight Majorana flavors. The interaction $H_{I}$ breaks the $\mathrm{SO}(8)$ symmetry down to its $\mathrm{SO}(7)$ subgroup and at the same time fixes $\chi_{a}$ to be one of the spinor representations $\mathbf{8}_{ \pm}$, say $\mathbf{8}_{+}$. Using the triality tensor $t_{a b}^{m}$, we can construct the physical fermion $\chi_{a}$ (as $\mathbf{8}_{+}$spinor) by fusing the bosonic parton $\phi_{b}$ (as $\mathbf{8}_{-}$spinor) and the fermionic parton $\psi_{m}$ (as 8 vector) on the field theory level:

$$
\chi_{Q a}=\sum_{b, m} t_{a b}^{m} \phi_{Q b} \psi_{Q m},
$$

which applies to both the left- and right-moving modes $Q=L, R$. One way to make sense of Eq. (C6) on the microscopic level is to consider the bosonic parton $\phi_{Q b}$ as a Kondo impurity resting on the boundary of the fermion chain [84], which is treated as a dynamical variable without spacial dependence. There are two types of Kondo impurities: $\phi_{L b}$ and $\phi_{R b}$. The $\phi_{Q b}$ impurity couples only to the $\chi_{Q a}$ fermion and scatters it to the $\psi_{Q m}$ fermion and vice versa. In this fractionalization scheme, both partons carry the $\mathrm{SO}(7) \subset \mathrm{SO}(8)$ symmetry charge, unlike the fractionalization scheme in the main text where only the bosonic parton carries the SU(4) symmetry charge. The translation and the chiral symmetry act on the parton fields as follows:

$$
T: \phi_{R b} \rightarrow-\phi_{R b}, \quad \mathcal{S}: \psi_{L m} \leftrightarrow \psi_{R m},
$$

such that the symmetry action on the physical fermion field in Eq. (C4) can be retrieved.

Now let us consider putting the fermionic parton $\psi_{m}=$ $\left(\psi_{L m}, \psi_{R m}\right)^{\top}$ in the same band structure as the physical fermion $\chi_{a}=\left(\chi_{L a}, \chi_{R a}\right)^{\top}$, described by

$$
\mathcal{L}_{0}^{\psi}=\frac{1}{2} \sum_{m=1}^{8} \bar{\psi}_{m} \gamma^{\mu} i \partial_{\mu} \psi_{m},
$$

similar to Eq. (C2). As long as the fermionic parton is gapless, the physical fermion is also gapless, which corresponds to the free-fermion fixed point. As the FK interaction is turned on, the following interaction for the fermionic parton will be induced:

$$
\mathcal{L}_{I}^{\psi}=-A\left(\sum_{m=1}^{7} \bar{\psi}_{m} \psi_{m}\right)^{2}-B\left(\sum_{m=1}^{7} \bar{\psi}_{m} \psi_{m}\right) \bar{\psi}_{8} \psi_{8},
$$

which contains two types of short-range interactions ( $A$ and $B$ terms). The general form of the parton interaction in Eq. (C9) can be argued on symmetry basis.
According to the fractionalization scheme Eq. (C6), the fermionic parton $\psi_{m}$ was assigned to the vector representation of $\mathrm{SO}(8)$. So the $\mathrm{SO}(7)$ symmetry group [as a subgroup of $\mathrm{SO}(8)$ ] will rotate only seven components of $\psi_{m}$ and leave one remaining component invariant. Without loss of generality, we assume $\psi_{8}$ to be the $\mathrm{SO}(7)$ invariant component, then the $\mathrm{SO}(7)$ vector can be written as $(m=1, \ldots, 7)$

$$
\Delta^{m}=i\left(\psi_{L m} \psi_{L 8}+\psi_{R m} \psi_{R 8}\right),
$$

in terms of the fermionic parton bilinear form. As shown in Eq. (C1), the FK interaction $H_{I} \sim-\boldsymbol{\Delta} \cdot \boldsymbol{\Delta}$ is just a dot product of $\mathrm{SO}(7)$ vectors, so we expect it to induce the same type of interaction for the fermionic partons

$$
-B \sum_{m=1}^{7} \Delta^{m} \Delta^{m}=-\frac{B}{2}\left(\sum_{m=1}^{7} \bar{\psi}_{m} \psi_{m}\right) \bar{\psi}_{8} \psi_{8} .
$$

This gives rise to the $B$-type of interaction in Eq. (C9). As shown in Ref. [19], under the RG flow, an $A$-type of interaction will be generated with $A>0$ and become relevant. The $A$-type interaction drives a spontaneous mass generation $\left\langle\sum_{m=1}^{7} i \bar{\psi}_{m} \psi_{m}\right\rangle=M$ for the first seven $\psi_{m}$ fermions, which in turn gives rise to the mass $i B M \bar{\psi}_{8} \psi_{8}$ for the $\psi_{8}$ fermion via the $B$-type interaction. Hence, all the fermionic partons are gapped out via the mass generation. The parton mass $M$ is evidently $\mathrm{SO}(7)$ symmetric. It is also invariant under the translation and the chiral symmetries as seen from Eq. (C7). So in the presence of the FK interaction, the system will enter the featureless gapped phase via spontaneous mass generation for the fermionic partons.

In conclusion, the key point that we learn from this $(1+1)$ D SMG is that the nature of the "symmetric mass" for the physical fermion is actually a bilinear mass for the fermionic parton. The fact that the parton bilinear mass does not break the symmetry is either because the fermionic parton is in a different symmetry representation from the physical fermion [e.g., the $\mathrm{SO}(7)$ symmetry] or because the symmetry charge is carried away by the bosonic parton (e.g., the translation symmetry). These are the key observations that motivate us to propose the $(2+1) \mathrm{D}$ SMG theory in the main text.

[1] P. R. Wallace, The Band Theory of Graphite, Phys. Rev. 71, 622 (1947).

[2] D. P. DiVincenzo and E. J. Mele, Self-Consistent EffectiveMass Theory for Intralayer Screening in Graphite Intercalation Compounds, Phys. Rev. B 29, 1685 (1984).

[3] S. Murakami, Phase Transition between the Quantum Spin Hall and Insulator Phases in 3D: Emergence of a Topological Gapless Phase, New J. Phys. 9, 356 (2007). 
[4] X. Wan, A. M. Turner, A. Vishwanath, and S. Y. Savrasov, Topological Semimetal and Fermi-Arc Surface States in the Electronic Structure of Pyrochlore Iridates, Phys. Rev. B 83, 205101 (2011).

[5] A. A. Burkov and L. Balents, Weyl Semimetal in a Topological Insulator Multilayer, Phys. Rev. Lett. 107, 127205 (2011).

[6] A. A. Burkov, M. D. Hook, and L. Balents, Topological Nodal Semimetals, Phys. Rev. B 84, 235126 (2011).

[7] S. M. Young, S. Zaheer, J. C. Y. Teo, C. L. Kane, E. J. Mele, and A. M. Rappe, Dirac Semimetal in Three Dimensions, Phys. Rev. Lett. 108, 140405 (2012).

[8] K. S. Novoselov, A. K. Geim, S. V. Morozov, D. Jiang, Y. Zhang, S. V. Dubonos, I. V. Grigorieva, and A. A. Firsov, Electric Field Effect in Atomically Thin Carbon Films, Science 306, 666 (2004).

[9] K. S. Novoselov, A. K. Geim, S. V. Morozov, D. Jiang, M. I. Katsnelson, I. V. Grigorieva, S. V. Dubonos, and A. A. Firsov, Two-Dimensional Gas of Massless Dirac Fermions in Graphene, Nature (London) 438, 197 (2005).

[10] Y. Zhang, Y.-W. Tan, H. L. Stormer, and P. Kim, Experimental Observation of the Quantum Hall Effect and Berry's Phase in Graphene, Nature (London) 438, 201 (2005).

[11] S. Borisenko, Q. Gibson, D. Evtushinsky, V. Zabolotnyy, B. Büchner, and R. J. Cava, Experimental Realization of a Three-Dimensional Dirac Semimetal, Phys. Rev. Lett. 113, 027603 (2014).

[12] H. Weng, C. Fang, Z. Fang, B. A. Bernevig, and X. Dai, Weyl Semimetal Phase in Noncentrosymmetric TransitionMetal Monophosphides, Phys. Rev. X 5, 011029 (2015).

[13] S.-M. Huang, S.-Y. Xu, I. Belopolski, C.-C. Lee, G. Chang, B. Wang, N. Alidoust, G. Bian, M. Neupane, C. Zhang, S. Jia, A. Bansil, H. Lin, and M. Z. Hasan, A Weyl Fermion Semimetal with Surface Fermi Arcs in the Transition Metal Monopnictide TaAs Class, Nat. Commun. 6, 7373 (2015).

[14] B. Q. Lv, H. M. Weng, B. B. Fu, X. P. Wang, H. Miao, J. Ma, P. Richard, X. C. Huang, L. X. Zhao, G. F. Chen, Z. Fang, X. Dai, T. Qian, and H. Ding, Experimental Discovery of Weyl Semimetal TaAs, Phys. Rev. X 5, 031013 (2015).

[15] S.-Y. Xu, I. Belopolski, N. Alidoust, M. Neupane, G. Bian, C. Zhang, R. Sankar, G. Chang, Z. Yuan, C.-C. Lee, S.-M. Huang, H. Zheng, J. Ma, D. S. Sanchez, B. Wang, A. Bansil, F. Chou, P. P. Shibayev, H. Lin, S. Jia, and M. Z. Hasan, Discovery of a Weyl Fermion Semimetal and Topological Fermi Arcs, Science 349, 613 (2015).

[16] A. H. Castro Neto, F. Guinea, N. M. R. Peres, K. S. Novoselov, and A. K. Geim, The Electronic Properties of Graphene, Rev. Mod. Phys. 81, 109 (2009).

[17] M. Franz and L. Molenkamp, Topological Insulators (Elsevier, New York, 2013), Vol. 6.

[18] D. J. Gross and A. Neveu, Dynamical Symmetry Breaking in Asymptotically Free Field Theories, Phys. Rev. D 10, 3235 (1974).

[19] L. Fidkowski and A. Kitaev, Effects of Interactions on the Topological Classification of Free Fermion Systems, Phys. Rev. B 81, 134509 (2010).

[20] L. Fidkowski and A. Kitaev, Topological Phases of Fermions in One Dimension, Phys. Rev. B 83, 075103 (2011).

[21] S. Ryu and S.-C. Zhang, Interacting Topological Phases and Modular Invariance, Phys. Rev. B 85, 245132 (2012).
[22] X.-L. Qi, A New Class of (2+1)-Dimensional Topological Superconductors With $\mathbb{Z}_{8}$ Topological Classification, New J. Phys. 15, 065002 (2013).

[23] H. Yao and S. Ryu, Interaction Effect on Topological Classification of Superconductors in Two Dimensions, Phys. Rev. B 88, 064507 (2013).

[24] L. Fidkowski, X. Chen, and A. Vishwanath, Non-Abelian Topological Order on the Surface of a 3D Topological Superconductor from an Exactly Solved Model, Phys. Rev. X 3, 041016 (2013).

[25] C. Wang and T. Senthil, Interacting Fermionic Topological Insulators/Superconductors in Three Dimensions, Phys. Rev. B 89, 195124 (2014).

[26] Z.-C. Gu and M. Levin, Effect of Interactions on TwoDimensional Fermionic Symmetry-Protected Topological Phases with $\mathrm{Z}_{2}$ Symmetry, Phys. Rev. B 89, 201113 (2014).

[27] Y.-Z. You and C. Xu, Symmetry-Protected Topological States of Interacting Fermions and Bosons, Phys. Rev. B 90, 245120 (2014).

[28] T. Yoshida and A. Furusaki, Correlation Effects on Topological Crystalline Insulators, Phys. Rev. B 92, 085114 (2015).

[29] Y. Gu and X.-L. Qi, Axion Field Theory Approach and the Classification of Interacting Topological Superconductors, arXiv:1512.04919.

[30] X.-Y. Song and A. P. Schnyder, Interaction Effects on the Classification of Crystalline Topological Insulators and Superconductors, Phys. Rev. B 95, 195108 (2017).

[31] R. Queiroz, E. Khalaf, and A. Stern, Dimensional Hierarchy of Fermionic Interacting Topological Phases, Phys. Rev. Lett. 117, 206405 (2016).

[32] Q.-R. Wang and Z.-C. Gu, Towards a Complete Classification of Fermionic Symmetry Protected Topological Phases in 3D and a General Group Supercohomology Theory, arXiv:1703.10937.

[33] M. A. Metlitski, L. Fidkowski, X. Chen, and A. Vishwanath, Interaction Effects on 3D Topological Superconductors: Surface Topological Order from Vortex Condensation, the 16 Fold Way and Fermionic Kramers Doublets, arXiv: 1406.3032 .

[34] To preserve the Dirac dispersion we avoid discussing Bernal stacked bilayer graphene. Instead, we have in mind twisted sheets of graphene that reduce interlayer tunneling.

[35] K. Slagle, Y.-Z. You, and C. Xu, Exotic Quantum Phase Transitions of Strongly Interacting Topological Insulators, Phys. Rev. B 91, 115121 (2015).

[36] I. Kimchi, S. A. Parameswaran, A. M. Turner, F. Wang, and A. Vishwanath, Featureless and Nonfractionalized Mott Insulators on the Honeycomb Lattice at 1/2 Site Filling, Proc. Natl. Acad. Sci. U.S.A. 110, 16378 (2013).

[37] S. Jiang and Y. Ran, Symmetric Tensor Networks and Practical Simulation Algorithms to Sharply Identify Classes of Quantum Phases Distinguishable by Short-Range Physics, Phys. Rev. B 92, 104414 (2015).

[38] P. Kim, H. Lee, S. Jiang, B. Ware, C.-M. Jian, M. Zaletel, J. H. Han, and Y. Ran, Featureless Quantum Insulator on the Honeycomb Lattice, Phys. Rev. B 94, 064432 (2016).

[39] Y. BenTov, Fermion Masses without Symmetry Breaking in Two Spacetime Dimensions, J. High Energy Phys. 07 (2015) 034. 
[40] Y.-Y. He, H.-Q. Wu, Y.-Z. You, C. Xu, Z. Y. Meng, and Z.-Y. Lu, Quantum Critical Point of Dirac Fermion Mass Generation without Spontaneous Symmetry Breaking, Phys. Rev. B 94, 241111 (2016).

[41] S. Catterall, Fermion Mass without Symmetry Breaking, J. High Energy Phys. 01 (2016) 121.

[42] V. Ayyar and S. Chandrasekharan, Origin of Fermion Masses without Spontaneous Symmetry Breaking, Phys. Rev. D 93, 081701 (2016).

[43] S.-S. Lee and P. A. Lee, U(1) Gauge Theory of the Hubbard Model: Spin Liquid States and Possible Application to $\kappa$-(BEDT-TTF $)_{2} \mathrm{Cu}_{2}(\mathrm{CN})_{3}$, Phys. Rev. Lett. 95, 036403 (2005).

[44] O. I. Motrunich, Variational Study of Triangular Lattice Spin-1/2 Model with Ring Exchanges and Spin Liquid State in $\kappa-(\mathrm{ET})_{2} \mathrm{Cu}_{2}(\mathrm{CN})_{3}$, Phys. Rev. B 72, 045105 (2005).

[45] D. N. Sheng, O. I. Motrunich, and M. P. A. Fisher, Spin Bose-Metal Phase in a Spin- $\frac{1}{2}$ Model with Ring Exchange on a Two-Leg Triangular Strip, Phys. Rev. B 79, 205112 (2009).

[46] M. S. Block, D. N. Sheng, O. I. Motrunich, and M. P. A. Fisher, Spin Bose-Metal and Valence Bond Solid Phases in a Spin-1/2 Model with Ring Exchanges on a Four-Leg Triangular Ladder, Phys. Rev. Lett. 106, 157202 (2011).

[47] S. Catterall and D. Schaich, Novel Phases in Strongly Coupled Four-Fermion Theories, Phys. Rev. D 96, 034506 (2017).

[48] V. Ayyar and S. Chandrasekharan, Fermion Masses through Four-Fermion Condensates, J. High Energy Phys. 10 (2016) 058.

[49] V. Ayyar, Search for a Continuum Limit of the PMS Phase, arXiv:1611.00280.

[50] T. Senthil, A. Vishwanath, L. Balents, S. Sachdev, and M.P.A. Fisher, Deconfined Quantum Critical Points, Science 303, 1490 (2004).

[51] O. I. Motrunich and A. Vishwanath, Emergent Photons and Transitions in the O(3) Sigma Model with Hedgehog Suppression, Phys. Rev. B 70, 075104 (2004).

[52] T. Senthil, L. Balents, S. Sachdev, A. Vishwanath, and M. P. A. Fisher, Quantum Criticality Beyond the LandauGinzburg-Wilson Paradigm, Phys. Rev. B 70, 144407 (2004).

[53] S. A. Kivelson, V. J. Emery, and H. Q. Lin, Doped Antiferromagnets in the Weak-Hopping Limit, Phys. Rev. B 42, 6523 (1990).

[54] E. Berg, E. Fradkin, and S. A. Kivelson, Theory of the Striped Superconductor, Phys. Rev. B 79, 064515 (2009).

[55] L. Radzihovsky and A. Vishwanath, Quantum Liquid Crystals in an Imbalanced Fermi Gas: Fluctuations and Fractional Vortices in Larkin-Ovchinnikov States, Phys. Rev. Lett. 103, 010404 (2009).

[56] E. Berg, E. Fradkin, and S. A. Kivelson, Charge-4e Superconductivity from Pair-Density-Wave Order in Certain High-Temperature Superconductors, Nat. Phys. 5, 830 (2009).

[57] E.-G. Moon, Skyrmions with Quadratic Band Touching Fermions: A Way to Achieve Charge 4e Superconductivity, Phys. Rev. B 85, 245123 (2012).

[58] Y.-F. Jiang, Z.-X. Li, S. A. Kivelson, and H. Yao, Charge-4e Superconductors: A Majorana Quantum Monte Carlo Study, Phys. Rev. B 95, 241103 (2017).
[59] Y.-Z. You and C. Xu, Interacting Topological Insulator and Emergent Grand Unified Theory, Phys. Rev. B 91, 125147 (2015).

[60] A. P. Schnyder, S. Ryu, A. Furusaki, and A. W. W. Ludwig, Classification of Topological Insulators and Superconductors in Three Spatial Dimensions, Phys. Rev. B 78, 195125 (2008).

[61] S. Ryu, A. P. Schnyder, A. Furusaki, and A. W. W. Ludwig, Topological Insulators and Superconductors: Tenfold Way and Dimensional Hierarchy, New J. Phys. 12, 065010 (2010).

[62] A. W. W. Ludwig, Topological Phases: Classification of Topological Insulators and Superconductors of NonInteracting Fermions, and Beyond, Phys. Scr. T168, 014001 (2016).

[63] C. Wu, Hidden Symmetry and Quantum Phases in Spin-3/2 Cold Atomic Systems, Mod. Phys. Lett. B 20, 1707 (2006).

[64] To preserve the fermion anticommutation relation, we may expect the bosonic parton $B_{i}$ to form a "unitary matrix": $\sum_{c} B_{i c a}^{\dagger} B_{i c b}=\sum_{c} B_{i a c} B_{i b c}^{\dagger}=\delta_{a b}$. However, this constraint turns out to be too strong, which restricts the boson Hilbert space to one dimension and effectively quenches all the bosonic degrees of freedom; i.e., there is only one state $\operatorname{det}\left(B_{i}^{\dagger}\right)\left|0_{B}\right\rangle$ that satisfies the constraint. So we choose to relax the constraint by allowing the boson number to fluctuate, meaning that we still keep the orthogonality but abandon the normalization of the $B_{i}$ matrix. With this, the physical fermion and the fermionic parton are still related by a unitary transform but up to a constant. The constant may be interpreted as the quasiparticle weight.

[65] Naively one may expect a larger U(4) gauge structure just by looking at the fractionalization scheme, but as we see soon, the interaction terms of the bosonic partons can break the gauge group down to $\mathrm{SU}(4)$. In a sense, we choose to fix the U(1) gauge.

[66] Note that the parton ground state $\left|\Psi_{f}\right\rangle$ is not gauge invariant, as it depends on the gauge sextet $\boldsymbol{M}$. This is legitimate because $\left|\Psi_{f}\right\rangle$ is not a physical state and $\boldsymbol{M}$ is not a variational parameter to appear in the final construction of the physical ground state after the gauge projection.

[67] Under the projective action of $\mathbb{Z}_{2}^{\mathcal{S}}$, the fermionic parton is particle-hole conjugated followed by the $\mathbb{Z}_{4}$ gauge transformation $f_{i}^{\dagger} \rightarrow-i(-)^{i} f_{i}$, and the parton vacuum state is sent to the fully occupied state $\left|0_{f}\right\rangle \rightarrow \prod_{i} f_{i 1}^{\dagger} f_{i 2}^{\dagger} f_{i 3}^{\dagger} f_{i 4}^{\dagger}\left|0_{f}\right\rangle$.

[68] T. Grover, Entanglement Monotonicity and the Stability of Gauge Theories in Three Spacetime Dimensions, Phys. Rev. Lett. 112, 151601 (2014).

[69] C. Vafa and E. Witten, Restrictions on Symmetry Breaking in Vector-like Gauge Theories, Nucl. Phys. B234, 173 (1984).

[70] Z. Cai, H.-h. Hung, L. Wang, D. Zheng, and C. Wu, Pomeranchuk Cooling of $S U(2 N)$ Ultracold Fermions in Optical Lattices, Phys. Rev. Lett. 110, 220401 (2013).

[71] Z. Cai, H.-H. Hung, L. Wang, and C. Wu, Quantum Magnetic Properties of the $S U(2 N)$ Hubbard Model in the Square Lattice: A Quantum Monte Carlo Study, Phys. Rev. B 88, 125108 (2013).

[72] D. Wang, Y. Li, Z. Cai, Z. Zhou, Y. Wang, and C. Wu, Competing Orders in the $2 D$ Half-Filled $S U(2 N)$ Hubbard 
Model through the Pinning-Field Quantum Monte Carlo Simulations, Phys. Rev. Lett. 112, 156403 (2014).

[73] Y.-Z. You, Z. Wang, J. Oon, and C. Xu, Topological Number and Fermion Green's Function for Strongly Interacting Topological Superconductors, Phys. Rev. B 90, 060502 (2014).

[74] V. Gurarie, Single-Particle Green's Functions and Interacting Topological Insulators, Phys. Rev. B 83, 085426 (2011).

[75] T. Senthil and M. P. A. Fisher, Competing Orders, Nonlinear Sigma Models, and Topological Terms in Quantum Magnets, Phys. Rev. B 74, 064405 (2006).

[76] C. Wang, A. Nahum, M. A. Metlitski, C. Xu, and T. Senthil, Deconfined Quantum Critical Points: Symmetries and Dualities, Phys. Rev. X 7, 031051 (2017).

[77] Y.-Z. You, Y.-C. He, A. Vishwanath, and C. Xu, From Bosonic Topological Transition to Symmetric Fermion Mass Generation, arXiv:1711.00863.

[78] J. Wang and X.-G. Wen, A Lattice Non-Perturbative Hamiltonian Construction of $1+1 \mathrm{D}$ Anomaly-Free Chiral
Fermions and Bosons-On the Equivalence of the Anomaly Matching Conditions and the Boundary Fully Gapping Rules, arXiv:1307.7480.

[79] X.-G. Wen, A Lattice Non-Perturbative Definition of an SO(10) Chiral Gauge Theory and Its Induced Standard Model, Chin. Phys. Lett. 30, 111101 (2013).

[80] Y.-Z. You, Y. BenTov, and C. Xu, Interacting Topological Superconductors and Possible Origin of $16 n$ Chiral Fermions in the Standard Model, arXiv:1402.4151.

[81] Y. BenTov and A. Zee, Origin of Families and SO (18) Grand Unification, Phys. Rev. D 93, 065036 (2016).

[82] C. Xu and S. Sachdev, Square-Lattice Algebraic Spin Liquid with SO(5) Symmetry, Phys. Rev. Lett. 100, 137201 (2008).

[83] C. Xu, Renormalization Group Studies on Four-Fermion Interaction Instabilities on Algebraic Spin Liquids, Phys. Rev. B 78, 054432 (2008).

[84] J. M. Maldacena and A. W. W. Ludwig, Majorana Fermions, Exact Mapping between Quantum Impurity Fixed Points with Four Bulk Fermion Species, and Solution of the Unitarity Puzzle, Nucl. Phys. B506, 565 (1997). 